\title{
The Companions of the Classical Cepheid FF Aql
}

\author{
NANCY REMAGE EVANS \& ANDREJ UDALSKI ${ }^{1}$ \\ Institute for Space and Terrestrial Science, York University, \\ c/o CRESS, 4700 Keele St., North York, M3J 1P3 Ontario, Canada
}

\begin{abstract}
We have obtained CCD photometry of the classical Cepheid FF Aql and the companion $6^{\prime \prime}$ away to determine whether they are likely to be physically associated. Because the Cepheid is 5 magnitudes brighter than the companion, the measurements are difficult. The values we find for the companion are $V=11.14 \pm$ $0.07 \mathrm{mag}, B-V=0.60 \pm 0.05 \mathrm{mag}$ and $V-R=0.39 \pm 0.04 \mathrm{mag}$. If the companion were at the same distance as the Cepheid, its absolute magnitude would be $M_{V}=$ $2.58 \mathrm{mag}$, corresponding a spectral type of approximately A8 V. The observed $(B-V)_{0}$ corresponds to a spectral type of F4 V to F5 V. The observed color and spectral type are incompatible with a ZAMS magnitude of the companion at the distance of the Cepheid.

Positional measures indicate that there may be relative motion between the two stars, which would be too large to be orbital motion. We conclude that the $6^{\prime \prime} .8 \mathrm{com}-$ panion is not a member of the FF Aql system. Information about the four stars in the direction of FF Aql is summarized.
\end{abstract}

\section{OBSERVATIONS}

The classical Cepheid FF Aql is a member of a complex system of up to four components. Modern techniques allow us to determine much more information about the components than Abt 1959 had available when he published the first spectroscopic orbit of the system. Specifically, in this paper we present the $V$ and $B-V$ of the companion $6^{\prime \prime}$ away, and discuss whether these data are consistent with a main sequence companion at the distance of the Cepheid. In the following section, we will summarize all the possible components of the system.

We (AU) observed FF Aql with the $61 \mathrm{~cm}$ telescope at the University of Toronto Las Campanas Observatory. Images were taken with the CCD system in $B, V$, and $R$ (Cousins). A fuller description of the observations and reductions is given by Udalski \& Evans (1992). The CCD has a plate scale of 0 ".45 per pixel. Because of the brightness of the Cepheid, each frame contained only the two stars, and reductions of the companion were done differentially with respect to the Cepheid. The Cepheid magnitude and colors at the phases of observation are known from previous light curves. Because of the large magnitude difference between the two stars and the small separation, it was difficult to accurately subtract the Cepheid light from the image of the companion. The approach finally taken was to create three artificial images rotated by $90^{\circ}, 180^{\circ}$, and $270^{\circ}$ from the original image and subtract the wing of the rotated Cepheid image from the companion image. The error of the mean result of all three subtractions (for all colors) was less than 0.05 mag. The resulting magnitudes and colors for the companion (transformed to the Cousins system) and also the data for the Cepheid are listed in Table 1.

\footnotetext{
${ }^{1}$ On leave from Warsaw University Observatory
} 
TABLE 1. Observed Magnitudes and Colors of the Visual Companion

\begin{tabular}{|c|ccc|cccc|}
\hline Date & \multicolumn{3}{|c|}{ Companion } & \multicolumn{4}{c|}{ Cepheid } \\
& $V$ & $(B-V)$ & $(V-R)_{C}$ & Phase & $V$ & $(B-V)$ & $(V-R)_{C}$ \\
\hline Sep 10, 1989 & 11.21 & 0.61 & 0.40 & 0.44 & $\mathbf{5 . 5 0}$ & $\mathbf{0 . 8 2}$ & 0.50 \\
Apr 3, 1990 & 11.13 & 0.55 & 0.39 & 0.37 & 5.47 & 0.80 & 0.50 \\
Apr 6, 1990 & 11.08 & 0.63 & 0.38 & 0.03 & 5.20 & 0.69 & 0.44 \\
\hline Average: & 11.14 & $\mathbf{0 . 6 0}$ & $\mathbf{0 . 3 9}$ & - & - & - & - \\
& \pm 0.07 & \pm 0.05 & \pm 0.04 & & & & \\
\hline
\end{tabular}

\section{DISCUSSION}

The next step is to compare the magnitude and colors with the values of main sequence stars at the distance of the Cepheid. Combining the absolute magnitude of the Cepheid from the period-luminosity-color relation of Feast \& Walker (1987) and the magnitude difference between the companion and the Cepheid (at mean light), the absolute magnitude of the companion is $2.58 \mathrm{mag}$, as listed in Table 2. Also listed in Table 2 is the $(B-V)_{0}$ of the companion, using the $\mathrm{E}(B-V)$ of the Cepheid $(0.18 \mathrm{mag})$. For comparison, values of $\mathrm{M}_{V}$ and $(B-V)_{0}$ for the ZAMS from Schmidt-Kaler (1982) are also listed in Table 2 for several spectral types. The $M_{V}$ for the companion at the distance of the Cepheid is too bright to be a ZAMS companion with the observed $(B--V)_{0}$, even allowing for reasonable errors in both the magnitude and colors of the companion.

TABLE 2. Absolute Magnitudes of FF Aql D and ZAMS Stars

\begin{tabular}{|cccc|}
\hline & $\begin{array}{c}\mathrm{M}_{V} \\
\text { mag }\end{array}$ & $\begin{array}{c}(B-V)_{0} \\
\text { mag }\end{array}$ & \\
\hline FF Aql D & 2.58 & 0.42 & \\
F0 V & 2.8 & 0.30 & Schmidt-Kaler \\
F2 V & 3.1 & 0.35 & Schmidt-Kaler \\
F4 V & 3.4 & 0.40 & Schmidt-Kaler \\
\hline
\end{tabular}

The parameters for the four stars in the direction of FF Aql system are summarized in Table 3, together with their sources. In the case of both the most distant companion and the speckle companion, there is possible motion between the two components which is too large to be Keplerian motion. Because of the relative motion and the CCD observations of the 6 ".8 companion, we conclude that it is not a member of the system. 
TABLE 3. Stars in the Direction of FF Aql

\begin{tabular}{|cccl|}
\hline Star & $\begin{array}{c}\text { Separation } \\
\mathrm{AU}\end{array}$ & $\begin{array}{c}\mathrm{V}_{0} \\
\text { mag }\end{array}$ & \multicolumn{1}{c|}{ Source } \\
\hline $\mathrm{A}$ & & 4.75 & Cepheid \\
$\mathrm{B}$ & 0.66 & & $\begin{array}{l}\text { Orbit: Abt (1959) } \\
\text { IUE: Evans et al. (1990) }\end{array}$ \\
$\mathrm{C}$ & 60 & $6:-7:$ & Speckle: McAlister et al. (1989) \\
D & 2650 & 10.5 & CCD: this paper \\
\hline
\end{tabular}

\section{ACKNOWLEDGMENTS}

We thank the Director of the Las Campanas Observatory for the use of the facility. This work was carried out with the financial support of Natural Science and Engineering Research Council (Canada) grants to N. R. Evans and S. M. Rucinski.

\section{REFERENCES}

Abt, H.A. $1959, A p J, 130,769$

Evans, N.R., Welch, D.L., Scarfe, C.D., \& Teays, T.J. 1990, AJ, 99, 1598

Feast, M.W. \& Walker, A.R. 1987, ARA\&A, 25, 345

McAlister, H.A., Hartkopf, W.I., Sowell, J.R., Dombrowski, E.G., \& Frans, O.G. 1989, $A J, 97,510$

Schmidt-Kaler, T. 1982, in Landolt-Börnstein VI 2b, edited by K. Schaifers and H. H. Voigt (Springer, New York), p. 18

Udalski, A. \& Evans, N.R. 1992, preprint 